\title{
National Inventory of Lymphoscintigraphy Procedure
}

\section{Mojgan Ebrahim and Rimma Axelsson}

\begin{abstract}
Mojgan Ebrahim
Division of Medical Imaging and Technology, Department of Clinical Science, Intervention and Technology (CLINTEC), Karolinska Institutet and Radiology department at Karolinska University Hospital, Huddinge, Stockhol, Sweden
\end{abstract}

Email: mojgan.ebrahim@ki.se

\begin{abstract}
Lymphoscintigraphy is a non-invasive technique for the evaluation of lymphatic function in patients with swollen legs. In spite of the fact that this technique has been used worldwide since the 1990s, there remains a lack of national or international procedure guidelines. The purpose of the study was to perform a Swedish national inventory on lymphoscintigraphic procedures for examining patients with swollen legs.
\end{abstract}

A survey was distributed to all 30 Swedish departments of nuclear medicine. The questionnaire comprised the number of lymphoscintigraphic examinations per year, the investigation procedure, and an evaluation of findings. The data obtained was analyzed and computed in terms of frequencies and diagrams using STATISTICA version 10.0.

There was an $86.6 \%$ response rate. Eleven respondents stated that they never performed lymphoscintigraphy, resulting in an analysis of 15 surveys. While the choice of radiotracer amount and type and site of injection varied between two different doses and compartments, the imaging time points were more inconsequent. Most respondents (73.3\%) avoided stocking during an imaging session, while only half of the clinics performed imaging in both passive and stressed conditions. The majority (93.3\%) of respondents used only visual lymphoscintigram interpretation, without the application of either a grading system or a quantitative evaluation.

This inventory indicates a national need for a standardized investigation procedure in patients with swollen legs. 


\section{INTRODUCTION}

Lymphoscintigraphy is used to obtain information about the morphology and function of the lymphatic system (Dąbrowski et al., 2008). It is considered an objective, non-invasive and reliable method to characterize the severity of lymphedema (Szuba, 2003) and to predict a therapeutic response (Walter et al., 2000; Yoo et al. 2014). In spite of the fact that this technique has been used worldwide since the 1990s, there remains a lack of guidelines from the European Association of Nuclear Medicine or the Northern America Society of Nuclear Medicine. The only available guidelines are published by the British Nuclear Medicine Society on their website, and aim to assist specialists in nuclear medicine in recommending, performing, interpreting and reporting the results of lymphoscintigraphy.

So far, every individual nuclear medicine department has formulated its local protocols. The purpose of the study was to perform a Swedish national inventory on lymphoscintigraphic procedures for examining patients with lower limb edema.

\section{MATERIAL AND METHODS}

A survey was distributed to all 30 Swedish hospitals with Nuclear Medicine departments. The questionnaire (Appendix 1) included 10 different questions regarding frequency of lymphoscintigraphic tests per year, administered radiotracer activity, residual measurement, type and site of injection, imaging times, passive/stress lymphoscintigraphy, quantitative/ qualitative evaluation of lymphoscintigraphy, support stockings, and also the purpose of the examination.

The data were analyzed and processed using the STATISTICA statistical program version
10.0 (StatSoft Inc., Tulsa, OK, USA). Descriptive statistics was used to summarize and organize the data.

\section{RESULTS}

Completed surveys were collected from 26 hospitals, which resulted in a response rate of $86.7 \%$.

\section{I) Frequency of lymphoscintigraphy}

This imaging technique was not used at all in 11 hospitals (42.3\%). Twelve hospitals (46.2\%) performed less than 10 lymphoscintigraphic investigations per year, and only three $(11.5 \%)$ performed more than 10 examinations per year.

\section{II) Administered radiotracer activity}

The radiolabelled colloids were administered as 40 or $80 \mathrm{MBq}$. The number of hospitals that used radiotracer with an activity of 40 $\mathrm{MBq}$ is only slightly greater than the number that used $80 \mathrm{MBq}(53.3 \%$ vs. $46.7 \%)$. There was no hospital in Sweden, which used radiotracer with an activity of less than 40 or greater than $80 \mathrm{MBq}$.

III) Support stocking requirements during the imaging procedure

Eleven centers $(73.3 \%)$ had clear procedures applicable to stockings so that elastic stockings, for lymphedema had to be removed during the whole imaging procedure, from the moment the patient entered the nuclear medicine department until going home. It was found that there were three centers $(20 \%)$ that had no support stocking procedures. These clinics performed lymphoscintigraphy to a lesser extent; up to 10 examinations per year. In one center, the patients removed their stockings only during, but not in-between, the imaging times. 
IV) Passive/stress lymphoscintigraphy

Eight of 15 centers performed acquisitions in both passive and stress conditions (Figure 1). Of these, only one center performed lymphoscintigraphy to a greater extent; up to 50 examinations a year.

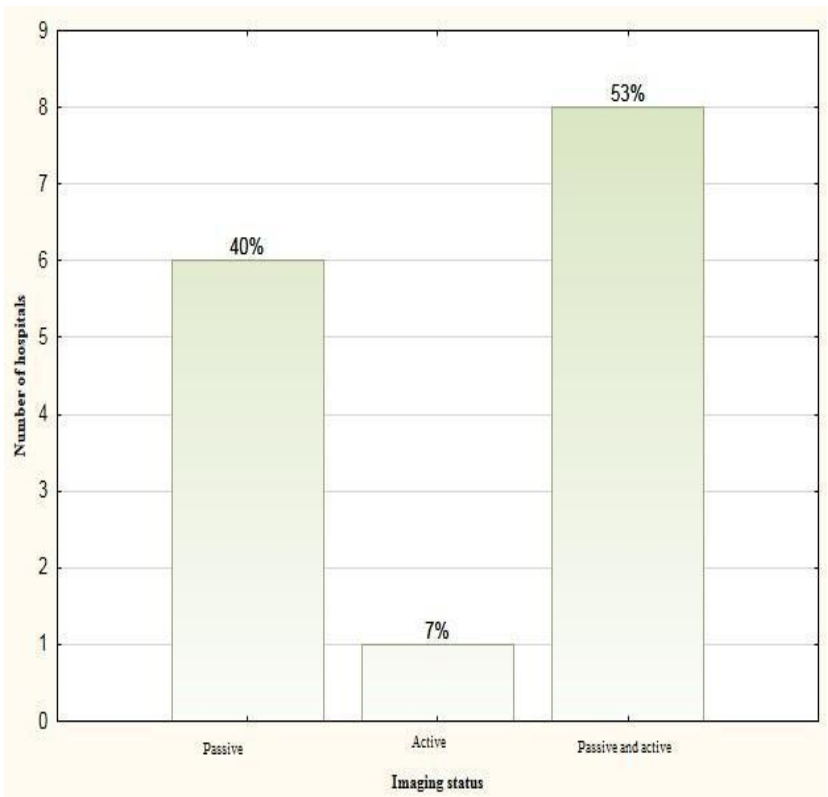

Figure 1: Frequency of passive and stress conditions during the lymphoscintigraphic technique

\section{V) Injection site}

The injection of radiotracer was administered both into the first and second web spaces of the foot in eight Swedish clinics. In three centers (20\%), the injection was performed only into the first web space of the foot and in four centers $(26.7 \%)$ into another location, for example, both into the first and third web spaces of the foot or dorsum of the foot.

\section{VI) Injection route}

Intradermal injection of radiotracer was performed in 10 Swedish clinics $(66.7 \%)$. In four centers $(26.7 \%)$ the injection was performed subcutaneously and, in one center (6.6\%), both injection techniques were used (Figure 2).

\section{VII) Residual measurements}

Four of 15 Swedish clinics performed residual measurements of syringe activity after injection to the lower limbs (Table 1). These clinics usually performed lymphoscintigraphy to a great extent; at least 50 examinations per year.

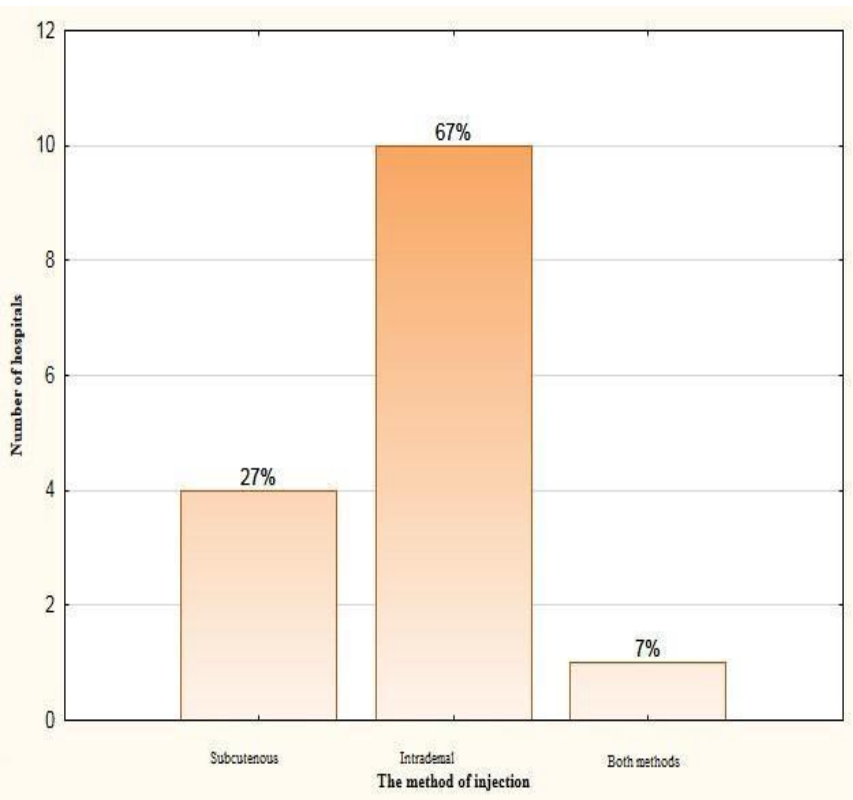

Figure 2: Frequencies of different injection methods

Table 1: Residual measurement frequencies

\begin{tabular}{|l|c|c|}
\hline $\begin{array}{l}\text { Residual } \\
\text { measurement }\end{array}$ & Yes & No \\
& $4(26.7 \%)$ & n $(\%)$ \\
\cline { 2 - 3 } & $11(73.3 \%)$ \\
\hline
\end{tabular}

VIII) Imaging time points

Acquisition times after injection varied greatly. Acquisitions began immediately post-injection in nine centers $(60 \%)$. In these clinics, an early image was acquired during the first hour after injection and a delayed image at the third and/or the fourth 
hour after injection. In one center, image acquisitions began a half-hour after injection and, in another, at 15 minutes post-injection and then at two hours after injection, with multiple images being acquired when needed. In one clinic, imaging times were five minutes after injection and then every 15 minutes during the first hour and then at the third hour as a delayed image. In another hospital, images were acquired at the first, second, third and fourth hours after injection. One center performed imaging at the sixth-hour postinjection and, eventually (if needed), the next day. In one hospital, imaging was performed at two-time points: once at one and a half hours and again at the third hour after injection.

\section{IX) Interpretation of lymphoscintigraphic studies}

The lymphoscintigraphic results were interpreted visually, e.g., qualitatively without the application of any quantification or grading system in almost all the hospitals $(93.3 \%)$ in Sweden, except one. This hospital used a combination of qualitative and quantitative interpretations of the images.

\section{X) Indications for using lymphoscintigraphy}

The method was mostly used in primary diagnosis to identify lymphedema in patients with swollen legs and rarely to assess post-therapeutic results. In 13 hospitals $(86.7 \%)$, lymphoscintigraphy was only used for primary diagnosis of the disease and in two hospitals $(13.3 \%)$ also for the evaluation of treatment results.

\section{DISCUSSION}

The response rate of $86.7 \%$ in our study is sufficient for the further evaluation of results and is in line with other studies.

\section{I) Frequency of lymphoscintigraphy}

Our survey shows that lymphoscintigraphy of the lower extremities is not common in Sweden: $88.5 \%$ of Swedish hospitals do not perform the lymphoscintigraphic examination, or the number of such examinations is fewer than 10 annually. A similar inventory was performed in the United Kingdom with a questionnaire sent to all members of the Vascular Society of Great Britain and Ireland. This showed that the majority of respondents (78\%) saw less than 10 new patients with lymphedema every year, and that lymphoscintigraphy was used to confirm the diagnosis by half of the respondents (Tiwari et al., 2006). It seems that diagnosis is, in certain sense, based on clinical examination and that lymphoscintigraphy is not likely done to alter the management plan.

Indeed, medical history and physical examination are mostly sufficient to give a correct diagnosis of the underlying lymphatic disorder, but imaging methods such as lymphoscintigraphy are necessary when a diagnosis of lymphedema is not clear, or other related diseases may disguise the relevance of lymphedema (International Society of Lymphology, 2009). Moreover, it has been suggested that this imaging method could be an essential part of the primary evaluation of any patient with suspected lymphedema (Lee et al., 2010). However, the results of our study show that about $40 \%$ of Swedish nuclear medicine departments do not perform this procedure at all, and only 3 of 30 departments perform more than 50 investigations per year.

On the other hand, there exists only a rough estimate of the incidence rate of lower limb lymphedema. For instance, about 2000 women were treated in 1994 for cancer of the genitals and lymphedema occurred in up to $40 \%$ of these women. Additionally 
approximately 7000 men were treated for prostate cancer and 5\% suffered lymphedema. The reported incidence of lymphedema is also very varied. The National Board of Health and Welfare records the different types of cases of disease in the Sweden but it has to first be sent the relevant diagnostic code. It is not uncommon for doctors to forget to write the diagnostic c for lymphedema in the journal and, therefore, the disease's statistics are inadequate and the real incidence rate is far higher than reported (Board of Swedish Edema Association, 2006).

\section{II) Administered radiotracer activity}

Both the uptake rate of the radiotracer in the lymph nodes and image quality can be affected by the amount of the radiotracer administered. As there is no national framework for the amount of radiotracer to be administered, every center creates its routines with the result that in some Swedish hospitals twice as many radiocolloids are administered as in other hospitals. The reported administered activity for imaging of the legs is approximately $0.5 \mathrm{mCi}$ or 18.5 $\mathrm{MBq}$ per extremity (Szuba et al., 2003; MacDonald \& Burrell, 2009), which has been used in the major older studies ((Weissleder \& Weissleder, 1998; Cambria et al., 1993), while an activity of about 40 $\mathrm{MBq}$ for each foot has been administered in more recent studies

(Dąbrowski et al., 2008; Damstra et al., 2008). According to the Administration of Radioactive Substances Advisory Committee (ARSAC) in the UK, the reference level for lymphoscintigraphic testing should be $20 \mathrm{MBq}$ per limb (BNMS Generic guidelines Lymphoscintigraphy, 2010). At present, the Swedish Radiation Safety Authority is attempting to harmonize administered activities in the most common imaging procedures in Sweden. This does not, however, include lymphoscintigraphy.

III) Support stocking requirements during the imaging procedure

One of the general criteria for lymphatic dysfunction is lymph diversion into the skin, resulting in the dermal backflow. The appearance of dermal backflow is pathognomonic for lymphedema. Use of support stockings will result in compression of the superficial system, with the outcome of preventing lymph from entering the dermal lymphatics (Keramida et al., 2014). Thus, keeping support stockings on would alter dermal back flow appearance during the lymphoscintigraphic imaging process] and would lead to false negative results. The absence of such a requirement is obviously insufficient and could lead to discrediting of the method. In most, but not all, Swedish centers $(73.3 \%)$, patients are asked to remove the elastic stockings for lymphedema during the whole investigation period, from the injection of radiocolloids to the last imaging session.

\section{IV) Passive/stress lymphoscintigraphy}

Lymphatic vessels do not contain muscles, and passive lymph flow can be slow. Exercise is thus able to intensify lymphatic drainage by extrinsic compression of lymphatic vessels by the skeletal musculature (BNMS Lymphoscintigraphy Generic Guidelines). Due to exercises the uptake of the particles into the lymphatics is substantially accelerated (Ikomi et al., 1995). Several studies have shown that the application of a stress procedure consequently increases the method's sensitivity (Szuba et al., 2003, Scarsbrook et 
al., 2007). Imaging after stress is therefore considered essential and is applied in several Swedish clinics as a routine method for the evaluation of the lymph system in patients.

\section{V) Injection site}

For the investigation of lower limb swelling, injections are usually performed into a web space. This is because tracer administered around the lateral foot and ankle may drain via the subfascial route, while tracer injected into a web space, under normal conditions, follows an epifascial route (Burnand et al., 2011). In Sweden, the radiotracer is mostly administered into the first and second web spaces of the lower extremities.

\section{VI) Injection route}

Both intradermal and subcutaneous injection is used to evaluate the epifascial system. Our inventory shows that the intradermal route is much more commonly used in the Swedish nuclear medicine centers. There are, however, conflicting views in the literature as to which route is better. Intradermal injection provides a larger surface area for tracer uptake with a more rapid uptake and clearance from the injection site, while subcutaneous injection is better at demonstrating the communication between subcutaneous tissues and the draining lymphatics (BNMS Lymphoscintigraphy / Generic Guidelines). On the other hand, intradermal injections are more difficult to carry out and there is a risk that some radiotracer will enter the subcutaneous space, thereby altering the results of the investigation (Keeley, 2006). In the United Kingdom, the subcutaneous route is recommended (BNMS Lymphoscintigraphy / Generic Guidelines).

\section{VII) Residual measurements}

Due to the adhesion/adsorption of the radiopharmaceutical to the syringe there is always resulting residual activity (dispensed activity - actually injected activity = residual activity). If the residual activity in the syringe is high, it can lead to the delivery of an inadequate dose to the patient that results in poor image quality or extended imaging times. There is, therefore, a requirement for a quality control program for the monitoring of residual activity in clinical practice (Swanson et al., 2013). However, residual measurements associated with lymphoscintigraphy of the lower extremities are performed in only $26.7 \%$ of Swedish hospitals.

\section{VIII) Imaging time points}

The imaging time points are more inconsequent, not only in Swedish clinics but also in the literature. There are several different instructions concerning when patients should be imaged after injection (Sadeghi et al., 2010; International Society of Lymphology, 2009; Yuan et al., 2006; Szuba et al., 2003). Two sets of images are usually acquired: a) early imaging up to one hour after injection, and b) delayed imaging at three or four hours post-injection. It has been reported that early images can be normal in some lymphedema patients and, taken alone, may give false negative results (Keeley, 2006). It is therefore recommended to take delayed images even with normal early images (Larcos \& Foster, 1995).

\section{IX) Interpretation of lymphoscintigraphic studies}

Quantitative lymphoscintigraphy is performed in only one nuclear medicine center in Sweden, but qualitative descriptive 
lymphoscintigraphy is much more common. Visual interpretation by qualitative lymphoscintigraphy is, in many cases, sufficient to provide a reliable diagnosis (Partsch, 2003). It may, however, possess lower sensitivity than a combination of quantitative and qualitative lymphoscintigraphy (Weissleder \& Weissleder, 1988; Dąbrowski et al., 2008). Although quantitative protocols for lymphoscintigraphy have shown excellent diagnostic performance, they have also been criticized because of an absence of a fixed reference area for lymph node activity after a given time interval (Jensen et al., 2010). Quantitative protocols have been used in some studies (Szuba et al., 2002; Kim et al., 2012) in order to assess the severity of lymphatic insufficiency. However, these studies have several limitations, including small sample size and complex methodologies which prevent clear application in clinical practice.

On the other hand, there is a lack of general agreement regarding the visual interpretation of lymphoscintigraphic findings. This method is based on a subjective assessment of the visual criteria and remains dependent on the experience of the interpreter (Jensen et al., 2010). Thus, there is a need for giving predetermined grades to some observed morphological features (e.g., the patterns of lymph flow, delay to flow and visualization of lymph nodes, flow through the deeper lymphatic system, and dermal diffusion) acquired by visual interpretation, which could show a good agreement between different investigators, e.g., through an interobserver study.

\section{X) Indications for using lymphoscintigraphy}

In Sweden, as well as in other European countries, lymphoscintigraphy of the lower extremities is performed for the differential diagnosis of edema (Burnand et al., 2011). However, this imaging technique could also be used to assess therapeutic results, and it is a potentially useful tool for the prognosis of long-term response to complex decongestive therapy (CDT) in patients with early-stage lymphedema (Yoo et al., 2014).

There are also other reported indications of lymphoscintigraphy in the literature such as the selection of patients for microsurgical operations (Campisi et al., 2003; Vaqueiro et al., 1986) and monitoring the efficacy of the operative procedure. In conclusion, this national inventory demonstrates the obvious discrepancies between the lymphoscintigraphic procedure for examining patients with swollen legs in the nuclear medicine centers in Sweden. It should also be emphasized that there is a need for the standardization of this imaging technique and the interpretation of results, which would promote a more appropriate application of this modality.

\section{REFERENCES}

Board of Swedish Edema Association. 2006. "Statistik över lymfödem" in the Swedish journal Lymfan, Volume 8 Number 2.

British Nuclear Medicine Society (BNMS) "Generic guidelines Lymphoscintigraphy".

(http://www.bnms.org.uk/images/stories/Pro cedures_and_Guidelines/Lymphoscintigraph y_wi th_referencesCF.pdf)

Burnand KM, Glass DM, Sundaraiya S, Mortimer PS, Peters AM. 2011, "Popliteal node visualization during standard pedal lymphoscintigraphy for a swollen limb indicates impaired lymph drainage. "AJR Am J Roentgenol 197(6):1443-8.

Campisi C, Boccardo F, Maccio A, Zilli A \& Schenone F. 2003. "Current use of microsurgery in lymphedema". 
Phlebolymphology. 43:216-219.

Dąbrowski J, Merkert R, Kuśmierek J. 2008. "Optimized lymphoscintigraphy and diagnostics of lymphatic oedema of the lower extremities". Nucl Med Rev Cent East Eur. 11(1):26-9.

Damstra RJ, van Steensel MA, Boomsma JH, Nelemans P, Veraart JC. 2008."Erysipelas as a sign of subclinical primary lymphoedema: a prospective quantitative scintigraphic study of 40 patients with unilateral erysipelas of the leg”. Br J Dermatol.158 (6):1210-5.

De Godoy JM, Lopes Pinto R, Pereira de Godoy AC, de Fátima Guerreiro Godoy M. 2014."Synergistic effect of adjustments of elastic stockings to maintain reduction in leg volume after mechanical lymph drainage". Int J Vasc Med. 640189.

Ikomi F, Hanna GK, Schmid-Schönbein GW.1995. "Mechanism of colloidal particle uptake into the lymphatic system: basic study with percutaneous lymphography". Radiology. 196(1):107-13.

International Society of Lymphology. 2009. "The diagnosis and treatment of peripheral lymphedema. 2009 Concensus Document of the International Society of Lymphology". Lymphology. 42(2):51-60.

Jensen MR1, Simonsen L, Karlsmark T, Bülow J. 2010."Lymphoedema of the lower extremities--background, pathophysiology and diagnostic considerations". Clin Physiol Funct Imaging. 30(6):389-98.

Keeley, V. 2006. "The use of lymphoscintigraphy in the management of chronic oedema".

Journal of lymphedema. 1(1): p. 42-57.

Keramida G, Humphrys M, Ryan N, Peters AM. 2014. "Stocking effect in lymphoscintigraphy". Lymphat Res Biol. 12(3):194-6.

Kim YB, Hwang JH, Kim TW, 2012. "Would complex decongestive therapy reveal long-term effect and lymphoscintigraphy predict thoutcome of lower-limb lymphedema related to gynecologic cancer treatment?' Gynecol Oncol 127:638-642.

Larcos G \& Foster DR. 1995. "Interpretation of lymphoscintigrams in suspected lymphoedema: contribution of delayed images". Nuclear Med Comm 16: 683-6

Lee B, Andrade M, Bergan J, Boccardo F, Campisi C, Damstra R, et al. International Union of Phlebology. 2010. "Diagnosis and treatment of primary lymphedema. Consensus document of the International Union of Phlebology (IUP)"- Int Angiol. 29(5):454-70.

MacDonald A, Burrell S. 2009. "Infrequently performed studies in nuclear medicine: part 2".

J Nucl Med Technol. 37(1):1-13.

Partsch H: 2003. "Practical aspects of indirect lymphography and lymphoscintigraphy".

Lymphat Res Biol. 1:71-73.

Sadeghi R, Kazemzadeh G, Keshtgar M. 2010. "Diagnostic application of lymphoscintigraphy in the management of lymphedema”. Hell J Nucl Med. 13(1):6-10.

Scarsbrook AF, Ganeshan A, Bradley KM. 2007."Pearls and pitfalls of radionuclide imaging of the lymphatic system. Part 2: evaluation of extremity lymphedema". Radiol. 280(951):219-26.

Swanson TN, Troung DT, Paulsen A, Hruska CB, O'Connor MK. 2013. "Adsorption of

99mTc-sestamibi onto plastic syringes: Copyright 2015 KEI Journals. All Rights Reserved 
evaluation of factors affecting the degree of adsorption and their impact on clinical studies". J Nucl Med Technol. 41(4):247-52.

Szuba A, Strauss W, Sirsikar SP. 2002." Quantitative radionuclide lymphoscintigraphy predicts outcome of manual lymphatic therapy in breast cancer-related lymphedema of the upper extremity". Nucl Med Commun. 23:11711175.

Szuba A, Shin WS, Strauss HW. 2003." The third circulation: Radionuclide lymphoscintigraphy in the evaluation of lymphedema”. J Nucl Med 44:43-57.

Tiwari A, Myint F, Hamilton G. 2006. "Management of lower limb lymphoedema in the United Kingdom". Eur J Vasc Endovasc Surg. 31(3):311-5.

Vaqueiro M, Gloviczki P, Fisher J, Hollier LH, Schirger A, Wahner HW. 1986.

"Lymphoscintigraphy in lymphedema: an aid to microsurgery". J Nucl Med. 27(7): 1125-30

Walter HW, Charles LW, Marlys HW. 2000.'Radionuclide lymphangioscintigraphy in the evaluation of peripheral lymphedema". Clin Nucl Med. 25:451-464.

Weissleder H, Weissleder R: 1998. "Lymphedema: Evaluation of qualitative and quantitative lymphoscintigraphy in 238 patients". Radiology. 167:729-735.

Yoo J, Choi JY, Hwang JH, Kim DI. 2014. "Prognostic value of lymphoscintigraphy in patients with gynecological cancer-related lymphedema”. J Surg Oncol. 109(8):760-3.

Yuan Z, Chen L, Luo Q, Zhu J, Lu H, Zhu R. 2006."The role of radionuclide lymphoscintigraphy in extremity lymphedema”. Ann Nucl Med. 20(5):341-4. 